\title{
How Family Physicians Address Diagnosis and Management of Depression in Palliative Care Patients
}

Franca Warmenhoven, $M D^{1}$

Eric van Rijswijk, $P b D^{1}$

Elise van Hoogstraten, $M D^{1}$

Karel van Spaendonck, $P b D^{1}$

Peter Lucassen, $P b D^{1}$

Judith Prins, $P h D^{2}$

Kris Vissers, $M D, P b D^{3}$

Chris van Weel, $M D, P b D^{1}$

'Department of Primary and Community Care, Radboud University Nijmegen Medical Centre, The Netherlands

${ }^{2}$ Department of Medical Psychology, Radboud University Nijmegen Medical Centre, The Netherlands

${ }^{3}$ Department of Anesthesiology, Pain and Palliative Medicine, Radboud University Nijmegen Medical Centre, The Netherlands

\begin{abstract}
PURPOSE Depression is highly prevalent in palliative care patients. In clinical practice, there is concern about both insufficient and excessive diagnosis and treatment of depression. In the Netherlands, family physicians have a central role in delivering palliative care. We explored variation in family physicians' opinions regarding the recognition, diagnosis, and management of depression in palliative care patients.
\end{abstract}

METHODS We conducted a focus group study in a sample of family physicians with varied practice locations and varying expertise in palliative care. Transcripts were analyzed independently by 2 researchers using constant comparative analysis in ATLAS.ti.

RESULTS In 4 focus group discussions with 22 family physicians, the physicians described the diagnostic and therapeutic process for depression in palliative care patients as a continuous and overlapping process. Differentiating between normal and abnormal sadness was viewed as challenging. The physicians did not strictly apply criteria of depressive disorder but rather relied on their clinical judgment and strongly considered patients' context and background factors. They indicated that managing depression in palliative care patients is mainly supportive and nonspecific. Antidepressant drugs were seldom prescribed. The physicians described difficulties in diagnosing and treating depression in palliative care, and gave suggestions to improve management of depression in palliative care patients in primary care.

CONCLUSIONS Family physicians perceive the diagnosis and management of depression in palliative care patients as challenging. They rely on open communication and a long-standing physician-patient relationship in which the patient's context is of great importance. This approach fits with the patient-centered care that is promoted in primary care.

Ann Fam Med 2012;10:330-336. doi:10.1370/afm.1373.

\section{INTRODUCTION}

Conflicts of interest: authors report none in previous 3 years.

\section{CORRESPONDING AUTHOR}

Franca Warmenhoven, MD

Department of Primary and Community

Care

Radboud University Nijmegen Medical

Centre

Huispost 117

PO Box 9101

6500 HB Nijmegen, The Netherlands

f.warmenhoven@anes.umcn.nl
M ood disturbances are common in palliative care patients. When confronted with a patient on a palliative trajectory who reports feeling sad, it can be challenging to differentiate depression from normal sadness. The prevalence of major depression and adjustment disorder in patients with advanced cancer is $6.7 \%$ and $16.3 \%$, respectively. ${ }^{1}$ Depression in palliative care is associated with poor treatment adherence, increased difficulties in relieving physical symptoms, disability, poor prognosis, higher mortality, longer inpatient stays, and elevated health care costs. ${ }^{2-6}$

Consequently, recognition, diagnosis, and treatment of depression are important. Health care professionals face the dilemma that active screening for and treatment of mood symptoms are encouraged, but may lead to overtreatment and medicalization of normal sadness..$^{7-9}$ In palliative care, this 
dilemma may be accentuated because mood symptoms can be related to the stress of facing terminal illness.

Palliative care is integral health care in which, in most countries, family physicians play an important role. ${ }^{10,11}$ Family physicians find it difficult to achieve balance between supporting and empowering patients with depressive symptoms, and actively treating patients with a depressive disorder. $9,12,13$ This difficulty could be even more prominent in palliative care, where physical symptoms and grief may hamper the interpretation of mood symptoms. ${ }^{5,14-16}$

The aim of this study was to explore the variation in the opinions of family physicians regarding the recognition, diagnosis, and management of depression in palliative care patients, and to ascertain their suggestions for optimizing this process.

\section{METHODS}

\section{Design and Setting}

The study took place in Dutch primary care. In the Netherlands, everyone is obliged to have private health care insurance, with all insurance plans providing care through family practice. Most palliative care patients want to die at home, with their family physician as the main professional caregiver. ${ }^{17}$

To study the perception of family physicians with regard to depression in palliative care patients, we used focus group discussions. A focus group study is considered an appropriate method to explore existing opinions about complex issues. ${ }^{18}$

\section{Participants}

We used purposive sampling to obtain a sample of family physicians from different locations and with varying expertise in palliative care. The sample consisted of 4 groups: (1) an existing continuing medical education group of physicians, discussing topics on a monthly basis; (2) a group of physicians whose practices were affiliated with the Radboud University for graduate studies in medicine $_{i}(3)$ a group of physicians of affiliated practices who had extensive expertise in palliative care; and (4) a group of physicians who were not affiliated with a university and whose practices were located in the South of the Netherlands. Group 1 had 8 physicians. The members of groups 2 and 3 were recruited by sending invitations to 300 family physicians; 61 physicians responded, of whom 8 were willing and able to participate in these groups. We selected the physicians with expertise in palliative care on the basis of participation in education, work, or research in this field Finally, of 24 physicians invited, 6 physicians were willing and able to participate in group 4 . All physicians were paid a modest fee for their participation.

\section{Questionnaire}

Each focus group participant answered a questionnaire on demographics, work experience, type of practice, religion, attitudes toward the use of screening instruments to diagnose depression, and the average number of palliative care patients they supported in 1 year. Additionally, using a $10-\mathrm{cm}$ visual analog scale, participants were asked to indicate their expertise in palliative care and in mental health; higher scores indicated greater expertise.

\section{Focus Groups}

An experienced, independent moderator (K.vS.) guided the focus group discussions with the topic list shown in Table 1. The topic list was developed from the literature and guidelines on depression in general and in palliative care. ${ }^{19-22}$ It focused on attitudes, problems, and needs in the management of depression in palliative care.

At the start of the focus group discussion, we gave a definition of a palliative care patient, describing
Table 1. Topic List for Focus Groups on Depression in Palliative Care Patients

Identification of depression in palliative patients

How do you recognize depression in a palliative patient?

Which specific complaints trigger you to think of depression?

What role do informal caregivers play in the assessment of depression?

How often do you recognize depression in a palliative patient?

Do you recognize or diagnose depression more easily in your palliative care patients after gaining more knowledge and experience in palliative care due to education and training? How would you describe the process of diagnosing depression in a palliative patient?

Do you use guidelines or standards in diagnosing depression in a palliative patient? What difficulties do you experience in diagnosing depression in a palliative patient? Have you had the experience where you missed a diagnosis of depression in a palliative patient or have recognized it (too) late?

Management of depressive symptoms in palliative patients

What is your general management for depression in palliative care patients?

What is your specific management for depression in palliative care patients?

Did your approach of depression in palliative care patients change after gaining more knowledge and experience in palliative care?

How do you perceive your role as a doctor in managing depression in palliative care patients? How are partners, spouses, and other close relatives of a palliative care patient involved in managing depression in palliative care patients?

Needs and solutions

What is needed in primary care to improve the identification and management of depressive complaints in the palliative phase? 
someone having a progressive, incurable illness, with a life expectancy of less than 1 year but more than a few weeks. We used this definition to direct the discussion to the care of patients on a palliative trajectory but not yet in the terminal phase of their illness.

We did not predefine depression because we wanted to observe physicians' implicit and explicit opinions on its definition. After briefly describing the group procedure, the moderator introduced each topic on the list, and each participant was invited to give his or her view. This step was followed by a group discussion in which the moderator actively motivated participants to explore and clarify their views more in depth. All focus group discussions were recorded and transcribed verbatim.

\section{Data Analysis}

We used constant comparative analysis to analyze the data. ${ }^{23,24}$ During this process, themes were continuously checked against the transcripts to ground the themes in the data using ATLAS.ti 4.2 (ATLAS.ti Scientific Software Development $\mathrm{GmbH}$ ).

The analysis started immediately after the first focus group and continued after each subsequent group. Two researchers (F.W. and E.vH.) independently coded the transcripts and categorized meaningful fragments to create structure in relevant themes. After each group, the codes were compared and discussed until agreement was reached. Subsequently, the codes were discussed with a third experienced researcher (E.vR.), to enhance rigor. We added important new themes to the topic list for consecutive focus groups until saturation was reached. ${ }^{18,25}$

\section{RESULTS}

\section{Participants and Procedure}

Our purposive sampling resulted in 4 focus groups having a total of 22 family physicians (13 men, 9 women, 4 to 8 participants per group). The physicians' characteristics are given in Table 2 . The focus group sessions lasted approximately 90 minutes and took place between February and April of 2010.

The results of the focus groups are split into 4 main categories: diagnosis of depression, management of depression, difficulties in this area, and needs and possible solutions. Below, we present different themes within each category.

\section{Diagnosis of Depression}

Depression Terminology, and Normal vs Pathologic Sadness

The family physicians reported that they regularly noticed a depressed mood, anxiety, sadness, and worry

\begin{tabular}{|c|c|}
\hline Characteristic & No. (\%) \\
\hline \multicolumn{2}{|l|}{ Sex } \\
\hline Male & $13(59)$ \\
\hline Female & $9(41)$ \\
\hline \multicolumn{2}{|l|}{ Employment status } \\
\hline Full time ${ }^{a}$ & $12(55)$ \\
\hline Part time & $10(45)$ \\
\hline \multicolumn{2}{|l|}{ Practice setting } \\
\hline Rural & $6(29)$ \\
\hline Suburban & $9(41)$ \\
\hline Urban & $6(29)$ \\
\hline \multirow{2}{*}{ Experience and expertise } & Mean (Range) \\
\hline & $18(3-33)$ \\
\hline Patients in their practice, No. & $4,575(1,850-7,300)$ \\
\hline Estimated palliative patients per year, No. & $11.5(3-20)$ \\
\hline VAS score ${ }^{b}$ expertise in palliative care, $\mathrm{cm}$ & $6.7(3.4-8.4)$ \\
\hline $\begin{array}{l}\text { VAS score }{ }^{\mathrm{b}} \text { expertise in mental health } \\
\text { care, } \mathrm{cm}\end{array}$ & $6.4(2.9-7.9)$ \\
\hline \multicolumn{2}{|l|}{ VAS = visual analog scale. } \\
\hline a Full time $=80 \%$ to $100 \%$ of full time. & values indicating greater \\
\hline
\end{tabular}

in palliative care patients, and generally felt competent to address these symptoms. They qualified emotional issues as understandable and appropriate in the process of accepting the end of life, and although they actively addressed these issues, they did not apply the medical concept of depression. They described difficulty in distinguishing normal from abnormal sadness, as the following comment illustrates:

I don't see it [depression] very often and I notice I have the tendency to ignore the medical concept [of depression] a little bit... I don't believe that at one point, a depressed mood starts and at another point, depressive disorder begins. There is of course a big transitional area, and it depends on the situation, the patient, and the doctor...I can't remember that I have ever actively looked for depression in a palliative patient (family physician [FP] D, focus group [FG] 1).

Interpreting Signs and Symptoms: Nature, Gravity, Consistency, and Expectations

The family physicians identified specific signs and symptoms that they related to depression: severe and persistent depressive feelings without moments of enjoyment or relief; flat affect; untreatable physical symptoms, or more suffering than would be expected from the medical condition; behavior that resulted in isolation and emotional distance from relatives and caregivers; a lack of growth toward accepting the end of life; and negative feelings or behavior that were not 
expected given the patient's character and background. As one physician stated:

The moment I think a person with cancer in this phase is depressed, then it [the behavior] has to differ from the pattern you expect there to be... and because you are a FP, you know how a patient normally reacts and whether it fits with the way I expect him to deal with this situation (FP H, FG 2).

The physicians reported that they often perceived the Diagnostic and Statistical Manual of Mental Disorders, Fourth Edition (DSM-IV) definition of depression as incompatible with the context of palliative care. They believed that the consistency and seriousness of the main symptoms of depression (depressive feeling and anhedonia) were important for diagnosis; however, they viewed additional criteria of depression, such as fatigue, weight loss, and anorexia, as inappropriate.

\section{The Diagnostic Process: Observation,}

Communication, Screening Tools, and "Gut Feeling" When asked how to diagnose depression in palliative care, the family physicians underscored the importance of mapping cognitions, social system, and the context of the patient to establish potentially changing patterns in feelings and behavior as a sign of psychological distress. To explore the character, seriousness, and origin of depressive feelings, the physicians reported that they discussed these aspects in open conversation with patients and, when relevant, also with partners and relatives.

They used the question "Do you feel depressed?" and saw it as useful for assessing the possibility of depression. Most physicians indicated that they relied on their clinical judgment and "gut feeling" rather than on screening instruments.

\section{Management of Depression}

\section{General Management: Context, Attention,} Communication, Support, and Autonomy

Whether or not the family physicians labeled patients as depressed, they stressed the importance of paying attention to depressive and anxious feelings; patients' $^{\prime}$ cognitions, existential questions, and spiritual needs; and the perceptions and feelings of partners. They reported that they attended to the psychosocial context early in the palliative phase to anticipate problems. Intensive support, frequent visits, and open communication were important tools in their management of depression. The physicians had observed that patients and families who felt that they could rely on their physician's support experienced less anxiety, more peace of mind, and decreased stress. Furthermore, physicians reported that they encouraged patients to make their own decisions in the palliative care trajectory as a means to increase their autonomy.
Specific Management, Referral, and Pharmacologic Treatment

Several family physicians reported that they sometimes found it helpful to refer patients to a psychologist, social worker, or palliative care team for support.

Physicians reported that they seldom prescribed antidepressants or stimulants, but those with greater expertise in palliative care indicated that they were more comfortable with diagnosing depression and deciding on pharmacologic treatment. The choice of medication was based on the nature of the psychological symptoms, the patient's age and life expectancy, comorbidities, and expected adverse effects.

Physicians who treated with antidepressants emphasized the importance of intensive support, especially in the beginning of the treatment, to increase treatment adherence. Pharmacologic treatment was always used in combination with psychotherapy or support.

\section{Difficulties in Providing Palliative Care}

\section{Interfering With the Natural Process}

The family physicians were reluctant to focus on depressive feelings out of fear of interfering with the process of patient adaptation, by negatively labeling it. Especially when it came to specific management, they expressed uncertainty. They often approached the possibility of depression indirectly with the patient, preserving a balance between focusing on patients' personal experiences and proactively managing depression. In view of this reservation, some physicians hesitated when it came to decisions about managing depressive symptoms with medication or psychotherapy. As one physician articulated, "I will not easily treat with antidepressants or diagnose depression because I don't want to interfere in the process of accepting the end of life" (FP V, FG 1).

\section{Context and Uniqueness of the Patient}

The family physicians emphasized the unique, dynamic situation of every patient that, in their opinion, made it difficult to follow general guidelines for diagnosis and management of depression, or even a specific guideline for depression in palliative care.

\section{Time, Knowledge, and Focus of Attention}

Time pressure was acknowledged as a structural barrier in palliative care in general and specifically in dealing with psychological issues. It limited conversations with patients and their partners.

Some physicians found it difficult to address the complete spectrum of palliative care, in particular, to discuss psychological or social aspects with patients and partners who were focused on the physical illness, as illustrated by the following comment: 
When you're very busy with all the somatic issues and symptoms and the management of the whole palliative care, the situation and everything around this, you don't have room to reflect calmly, like, "How was the patient doing, what was the interaction, and can there be a depressive undertone?" (FP D, FG 3).

\section{Needs and Possible Solutions}

The family physicians noted shortcomings in the care for depression in patients on a palliative trajectory and indicated they would have liked to have more education, especially on identifying depression in palliative care, or support from a guideline, or access to questionnaires or criteria to distinguish between normal and abnormal depressive feelings.

Physicians with expertise in palliative care stressed the importance of having a routine when it came to the technical and logistical management of this care, to create time for psychosocial care.

They pointed to the lack of collaboration with other disciplines, and lack of insight of those disciplines' possible contributions. They noted that promoting teamwork with family physicians, specialists in hospitals, therapists, palliative care or nursing teams, social workers, priests, or imams was valuable and might result in more attention to patients' psychological, emotional, social, and spiritual needs. Furthermore, they saw a larger role for mental health professionals who are trained to work with palliative care patients and their partners; as one physician commented, "I notice that there are few places where I can refer my [palliative care] patients to" (FP H, FG 2).

\section{DISCUSSION}

The family physicians in this study reported that they were frequently confronted with depressive symptoms in their palliative care patients and in general felt competent to address this issue.

In supporting these patients, the physicians heavily relied on cumulative knowledge in determining whether patients had normal sadness or depression that required an intervention. They acknowledged the difficulty of discerning depression from normal sadness and sometimes experienced a lack of knowledge, time, and additional support sources, such as specialized psychologists.

To enhance management of depression in palliative care patients, family physicians suggested (1) specific attention to the diagnostic process of depression in palliative care patients in their professional education, (2) a specific and practical guideline for depression in palliative care, (3) a psychometric tool to assess depression in palliative care patients and to follow up on treatment, (4) the availability of mental health professionals trained in palliative care, and (5) enhanced collaboration with other health care professionals.

The physicians reported that they valued their clinical judgment more than questionnaires. Other studies have similarly found that family physicians consider their practical wisdom and clinical judgment to be more important than objective assessments. ${ }^{7}$ In this context, depression instruments can aid in shared decision making and monitoring. ${ }^{26}$

Most family physicians did not often explicitly diagnose a depressive disorder in palliative care patients. In fact, one physician expressed fear about interfering with the process of accepting the end of life by labeling sadness as pathologic. These findings contrast with those of an earlier study of depressed patients in primary care in which family physicians encouraged patients to view depression as separate from the self and normal sadness. ${ }^{27}$ This difference between studies illustrates the specific context of palliative care patients requiring a different approach. A disadvantage of a conservative approach to diagnosing depression might be that depressed palliative care patients do not receive optimal treatment; however, if the supportive and contextual care that family physicians offer is adequate for most patients, it might spare many patients unnecessary intensive and sometimes harmful treatment.

Several palliative care guidelines call for proactive screening for and treatment of depression in patients with terminal cancer who have a limited survival time. ${ }^{28,29}$ Models for active screening and treatment of mood symptoms are often based on the medical model, however, and that model does not consider family physicians' care to be the most adequate approach to addressing mood symptoms in palliative care. This situation might explain why some family physicians do not actively look for depression in palliative care patients, even though they are aware that depressive symptoms are quite common in this population. Family physicians seem to be more interested in clinical utility than in the validity of the diagnosis of depression. ${ }^{30-32}$ They use a contextual concept of depression, which may reflect the patient-centered and context-specific care that is promoted in family practice. ${ }^{33,34}$ This viewpoint was also reflected in the comments of participants who maintained that because of a long-term relationship with patients, they were able to determine whether the patient's behavior was consistent with the pattern they would expect in the expression of normal sadness or whether it was consistent with pathologic sadness.

From the perspective of patient-centered care, it might prove helpful if family physicians spoke openly with their patients and caregivers about how to relate to sadness in the process of facing terminal illness in 
the patients' unique situation. Family physicians could inform their palliative care patients that depression is sometimes difficult to assess in the context of a palliative trajectory and that it needs monitoring.

Patients and caregivers could be encouraged to express how they value their sadness and whether they feel their sadness is normal or abnormal. Furthermore, the physicians should discuss with the patient and caregivers treatment options for depression, and both possible benefits and burdens in relation to the specific context of the patient. Practical advice for both patient and caregivers (such as seeking distraction and pleasant activities, approaching the patient not only in the patient role) can also be important.

To our knowledge, this is the first study to explore how family physicians address depression in palliative care. We used a purposive sampling strategy to reach an optimal variety of opinions. The limited number of physicians may limit extrapolation of the results to all family physicians but is considered adequate for a first in-depth exploration. ${ }^{35}$ Although the study reflects the Dutch primary care context, we are confident that our findings are valuable more generally: first and foremost, the importance of palliative care, in or as close as possible to the patient's own environment, is a value shared by family physicians from many countries. In delivering care, the physicians in this study referred to their professional relationship with patients and their use of knowledge of patients and their family accumulated over time, which are core values of international primary care. ${ }^{36}$

In focus group discussions, participants influence each other, therefore, contrasting opinions and results may be silenced. ${ }^{35}$ In this study, however, we found that all participants seemed to share their views openly, and we obtained a variety of opinions. We included group discussions with participants who did not know each other and group discussions with established groups in which members might be more used to challenging each other's views and opinions.

Although the moderator provided a broad working definition of a palliative care patient, almost all participants seemed to confine the discussion to patients with cancer. In future research, we recommend explicitly including in the discussion palliative care patients who do not have cancer. Family physicians might have a different approach to depression in this population. ${ }^{37}$

Two of the researchers who analyzed the data (F.W. and E.vR.) are family physicians and familiar with the practice of family medicine, and therefore are more likely to interpret the data in the appropriate context.

Finally, the opinions and experiences that the family physicians expressed and described do not provide data about the physicians' actual behavior and the effects on patient outcomes.
In conclusion, family physicians play a pivotal role in providing patient-centered and context-specific mental health care for palliative care patients. On the basis of the findings of this study, we suggest that family physicians acknowledge the importance of the diagnosis and management of depression in palliative care and monitor patients' psychological well-being over time. We recommend improving family physicians' education by building on the elements that our study participants presented in diagnosing depression and distinguishing it from normal sadness: strengthening continuity of care and relationship building with patients and their families, throughout the palliative trajectory, and explicitly addressing sadness as part of the normal process of coming to terms with the prospect of the end of life. Guidelines, criteria, and patient-friendly psychometric tools will provide valuable support only when applicable in the context of such a person-centered approach. In this way, family physicians can be supported to further improve their approach to the complex situation of palliative care patients facing challenges in nearly all domains (physical, psychological, spiritual, and social). This approach acknowledges the importance and use of cumulative and contextual knowledge, which is a core quality of family practice. ${ }^{38}$

Finally, more research is needed to evaluate the actual diagnostic and therapeutic processes that family physicians use in managing depression in palliative care and the effects on their patients.

To read or post commentaries in response to this article, see it online at http://www.annfammed.org/content/10/4/330.

Key words: family practice; depressive disorder; depression; palliative care; focus group; practice-based research

Submitted May 6, 2011; submitted, revised, September 29, 2011; accepted October 17, 2011.

\section{References}

1. Akechi T, Okuyama T, Sugawara $Y$, Nakano $T$, Shima $Y$, Uchitomi $Y$. Major depression, adjustment disorders, and post-traumatic stress disorder in terminally ill cancer patients: associated and predictive factors. J Clin Oncol. 2004;22(10):1957-1965.

2. Irving G, Lloyd-Williams M. Depression in advanced cancer. Eur J Oncol Nurs. 2010;14(5):395-399.

3. Lloyd-Williams M, Friedman T, Rudd N. A survey of antidepressant prescribing in the terminally ill. Palliat Med. 1999;13(3):243-248.

4. Lloyd-Williams M, Shiels C, Taylor F, Dennis M. Depression-an independent predictor of early death in patients with advanced cancer. J Affect Disord. 2009;113(1-2):127-132.

5. Rayner L, Loge JH, Wasteson E, Higginson IJ; EPCRC, European Palliative Care Research Collaborative. The detection of depression in palliative care. Curr Opin Support Palliat Care. 2009;3(1):55-60.

6. Reeve JL, Lloyd-Williams M, Dowrick C. Revisiting depression in palliative care settings: the need to focus on clinical utility over validity. Palliat Med. 2008;22(4):383-391. 
7. Dowrick C, Leydon GM, McBride A, et al. Patients' and doctors' views on depression severity questionnaires incentivised in UK quality and outcomes framework: qualitative study. BMJ. 2009;338:b663

8. Horwitz AV, Wakefield JC. The Loss of Sadness: How Psychiatry Transformed Normal Sorrow Into Depressive Disorder. New York, NY: Oxford University Press; 2007.

9. van Rijswijk $E$, van Hout $H$, van de Lisdonk $E$, Zitman $F$, van Weel $C$. Barriers in recognising, diagnosing and managing depressive and anxiety disorders as experienced by family physicians; a focus group study. BMC Fam Pract. 2009;10:52.

10. Aabom B, Kragstrup J, Vondeling H, Bakketeig LS, Stovring H. Does persistent involvement by the GP improve palliative care at home for end-stage cancer patients? Palliat Med. 2006;20(5):507-512.

11. Neergaard MA, Vedsted $P$, Olesen F, Sokolowski I, Jensen AB, Sondergaard J. Associations between successful palliative trajectories, place of death and GP involvement. Scand J Prim Health Care. 2010;28(3):138-145.

12. Andersson SJ, Troein M, Lindberg G. Conceptions of depressive disorder and its treatment among 17 Swedish GPs. A qualitative interview study. Fam Pract. 2001;18(1):64-70.

13. Chew-Graham CA, Mullin S, May CR, Hedley S, Cole H. Managing depression in primary care: another example of the inverse care law? Fam Pract. 2002;19(6):632-637.

14. Delgado-Guay M, Parsons HA, Li Z, Palmer JL, Bruera E. Symptom distress in advanced cancer patients with anxiety and depression in the palliative care setting. Support Care Cancer. 2009;17(5):573-579.

15. Mystakidou K, Parpa E, Tsilika E, et al. Preparatory grief, psychological distress and hopelessness in advanced cancer patients. Eur J Cancer Care (Engl). 2008;17(2):145-151.

16. Periyakoil VS, Hallenbeck J. Identifying and managing preparatory grief and depression at the end of life. Am Fam Physician. 2002; 65(5):883-890.

17. van der Akker P, Luijkx K, van Wersch S. Wetenschappelijk Rapport: Waar wilt u doodgaan? Keuzen en overwegingen. 2005. http:// www.vptz.nl/dynamic/media/1/documents/wetensch_rapport_waar wilt_u_doodgaan.pdf. Accessed June 10, 2012.

18. Kreuger RA, Casey MA. Focus Groups. A Practical Guide for Applied Research. 3rd ed. Thousand Oaks, CA: Sage Publications; 2000.

19. NHG Standaard Depressieve Stoornis. 2010. http://depressie.wikidot.com/nhg-standaard-depressieve-stoornis. Accessed June 10, 2012.

20. Bannink M, Monster JC. Richtlijn Depressie versie 2.0. In: Richtlijnenboek Palliatieve zorg. The Netherlands: Vereniging van Integrale kankercentra; 2010.

21. Hotopf M, Chidgey J, Addington-Hall J, Ly KL. Depression in advanced disease: a systematic review Part 1. Prevalence and case finding. Palliat Med. 2002;16(2):81-97.

22. Ly KL, Chidgey J, Addington-Hall J, Hotopf M. Depression in palliative care: a systematic review. Part 2. Treatment. Palliat Med. 2002;16(4):279-284.
23. Cutcliffe JR. Adapt or adopt: developing and transgressing the methodological boundaries of grounded theory. J Adv Nurs. 2005; 51(4):421-428.

24. Strauss AL. Qualitative Analysis for Social Scientists. Oxford, England: Cambridge University Press; 1987.

25. Britten N. Qualitative interviews in medical research. BMJ. 1995; 311(6999):251-253.

26. Baik SY, Gonzales JJ, Bowers BJ, Anthony JS, Tidjani B, Susman JL. Reinvention of depression instruments by primary care clinicians. Ann Fam Med. 2010;8(3):224-230

27. Johnston O, Kumar S, Kendall K, Peveler R, Gabbay J, Kendrick T. Qualitative study of depression management in primary care: GP and patient goals, and the value of listening. Br J Gen Pract. 2007;57(544):872-879.

28. Qaseem A, Snow V, Shekelle P, et al; Clinical Efficacy Assessment Subcommittee of the American College of Physicians. Evidence-based interventions to improve the palliative care of pain, dyspnea, and depression at the end of life: a clinical practice guideline from the American College of Physicians. Ann Intern Med. 2008;148(2):141-146.

29. Stiefel R, Die Trill M, Berney A, Olarte JM, Razavi A. Depression in palliative care: a pragmatic report from the Expert Working Group of the European Association for Palliative Care. Support Care Cancer. 2001;9(7):477-488.

30. Dowrick C. When diagnosis fails: a commentary on McPherson $\&$ Armstrong. Soc Sci Med. 2009;69(8):1144-1146.

31. McPherson S, Armstrong D. Negotiating 'depression' in primary care: a qualitative study. Soc Sci Med. 2009;69(8):1137-1143.

32. van Weel C, van Weel-Baumgarten E, van Rijswijk E. Treatment of depression in primary care. BMJ. 2009;338:b934.

33. Epstein RM, Street RL Jr. The values and value of patient-centered care. Ann Fam Med. 2011;9(2):100-103.

34. Lloyd-Williams M, Reeve J, Kissane D. Distress in palliative care patients: developing patient-centred approaches to clinical management. Eur J Cancer. 2008:44(8):1133-1138.

35. Kitzinger J. Qualitative research. Introducing focus groups. BMJ. 1995;311(7000):299-302

36. Allen J, Gay B, Crebolder H, Heyrman J, Svab I, Ram P. The European Definition of General Practice/Family Medicine. European Academy of Teachers in General Practice Council. Evans P, ed. Prepared for WONCA Europe; 2005. http://www.woncaeurope.org/ content/european-definition-general-practicefamily-medicine-2005. Accessed June 10, 2012.

37. Patten SB, Williams JV, Lavorato $\mathrm{DH}$, Modgill G, Jetté N, Eliasziw M Major depression as a risk factor for chronic disease incidence: longitudinal analyses in a general population cohort. Gen Hosp Psychiatry. 2008;30(5):407-413.

38. Burroughs H, Lovell K, Morley M, Baldwin R, Burns A, Chew-Graham C. 'Justifiable depression': how primary care professionals and patients view late-life depression? A qualitative study. Fam Pract. $2006 ; 23(3): 369-377$. 